\title{
Theory of neutrino emission from nucleon-hyperon matter in neutron stars: Angular integrals
}

\author{
A. D. Kaminker ${ }^{1}$ • D. G. Yakovlev ${ }^{1}$ • P. Haensel ${ }^{2}$
}

\begin{abstract}
Investigations of thermal evolution of neutron stars with hyperon cores require neutrino emissivities for many neutrino reactions involving strongly degenerate particles (nucleons, hyperons, electrons, muons). We calculate the angular integrals $I_{n}$ (over orientations of momenta of $n$ degenerate particles) for major neutrino reactions with $n=3,4,5$ at all possible combinations of particle Fermi momenta. The integrals $I_{n}$ are necessary ingredients for constructing a uniform database of neutrino emissivities in dense nucleon-hyperon matter. The results can also be used in many problems of physical kinetics of strongly degenerate systems.
\end{abstract}

Keywords Strongly degenerate fermions, reaction rates, angular integration

\section{Introduction}

It is well known that thermal evolution of not too cold neutron stars is regulated by the neutrino emission from superdense matter in neutron star cores. In order to model the thermal evolution one needs the emissivies of many neutrino reactions which can operate and produce an efficient neutrino cooling of these stars (e.g., Yakovlev et al. 2001). Consider, for instance, neutron star cores, which are massive and bulky internal regions of neutron stars (Shapiro and Teukolsky 1983). They are thought to contain uniform nuclear liquid of

\footnotetext{
A. D. Kaminker

D. G. Yakovlev

P. Haensel

${ }^{1}$ Ioffe Physical Technical Institute, Politekhnicheskaya 26, 194021, St Petersburg, Russia

${ }^{2}$ N. Copernicus Astronomical Center, Bartycka 18, 00-716 Warsaw, Poland
}

density $\rho$ ranged from $\sim \rho_{0} / 2$ to $\sim 10-20 \rho_{0}$, where $\rho_{0} \approx 2.8 \times 10^{14} \mathrm{~g} \mathrm{~cm}^{-3}$ is the density of standard nuclear matter at saturation. A neutron star core can be divided into the outer core $\left(\rho \lesssim 2 \rho_{0}\right)$ composed of neutrons $(\mathrm{n})$ with some admixture of protons $(\mathrm{p})$, electrons (e) and muons $(\mu)$, and the inner core $\left(\rho \gtrsim 2 \rho_{0}\right)$ containing the same particles and possibly other ones (for instance, hyperons). All constituents of the matter (n, p, e, $\mu$, hyperons) are strongly degenerate fermions. These particles can participate in many reactions producing neutrinos. Because in half a minute after their birth neutron stars become fully transparent for neutrinos, the neutrinos immediately escape from the star and cool it.

Schematically, the neutrino emissivity $\left[\mathrm{erg} \mathrm{cm}^{-3} \mathrm{~s}^{-1}\right.$ ] for any reaction can be written as

$Q=(2 \pi)^{4} \int \mathrm{d} \Gamma \mathcal{M}_{\mathcal{F} \mathcal{I}} \epsilon_{\nu} \delta\left(\boldsymbol{P}_{\mathcal{F}}-\boldsymbol{P}_{\mathcal{I}}\right) \delta\left(E_{\mathcal{F}}-E_{\mathcal{I}}\right) F_{\mathcal{F} \mathcal{I}}$

Here, $\epsilon_{\nu}$ is the energy of generated neutrino (or neutrinos), $\mathcal{I}$ and $\mathcal{F}$ label initial and final states of a system, while $i$ and $f$ label corresponding states of reacting particles; $\boldsymbol{P}_{\mathcal{I}}=\sum_{i} \boldsymbol{p}_{i}$ and $\boldsymbol{P}_{\mathcal{F}}=\sum_{f} \boldsymbol{p}_{f}$ denote, respectively, total momenta of reacting particles in the $\mathcal{I}$ and $\mathcal{F}$ states, with $\boldsymbol{p}$ being a one-particle momentum; $E_{\mathcal{I}}=\sum_{i} \epsilon_{i}$ and $E_{\mathcal{F}}=\sum_{f} \epsilon_{f}$ are total energies of the particles ( $\epsilon$ is a one-particle energy). The delta functions take into account momentum and energy conservation in a reaction event. The factor $F_{\mathcal{F} \mathcal{I}}$ is

$$
\begin{aligned}
F_{\mathcal{F} \mathcal{I}} & =\left(\prod_{i} f_{i}\right)\left(\prod_{f}\left(1-f_{f}\right)\right), \\
f_{i} & =\left[\exp \left(\frac{\epsilon_{i}-\mu_{i}}{k_{\mathrm{B}} T}\right)+1\right]^{-1} .
\end{aligned}
$$


It contains the product of Fermi-Dirac functions $f_{i}$ for particles in the initial states and the product of blocking functions $\left(1-f_{f}\right)$ for particles in the final states; $\mu_{i}$ is the chemical potential, $T$ the temperature, and $k_{\mathrm{B}}$ the Boltzmann constant. In what follows, we take into account that neutron star matter is fully transparent for neutrinos (e.g., Yakovlev et al. 2001). Then the chemical potential of neutrinos is zero, $\mu_{\nu}=0$, and the approximation of massless neutrinos is excellent. Other initial or final reacting fermions $j$ (which belong to the dense matter) are assumed to be strongly degenerate particles of any relativity; their energies $\epsilon_{j}$ and chemical potentials $\mu_{j}$ may include or exclude the restmass energy, $m_{j} c^{2}$. The quantity $\mathcal{M}_{\mathcal{F} \mathcal{I}}$ in Eq. (1) is proportional to the squared matrix element for a given reaction summed over spin states. Finally,

$\mathrm{d} \Gamma=\prod_{l} \frac{\mathrm{d} \boldsymbol{p}_{l}}{(2 \pi \hbar)^{3}}$

is the product of densities of states of all reacting particles $l=j$ and $\nu$.

It is well known (e.g., Ziman 1960; Baym and Pethick 2007; Shapiro and Teukolsky 1983) that calculation of the emissivities (1), reaction rates or related quantities in a strongly degenerate matter is greatly simplified because the main contribution into corresponding integrals comes from narrow thermal energy widths $\mid \epsilon_{j}-$ $\mu_{j} \mid \ll k_{\mathrm{B}} T$. Accordingly, one can usually employ the so called energy-momentum decomposition detailed, e.g., in Ziman (1960); Baym and Pethick (2007); Shapiro and Teukolsky (1983). It consists in fixing lengths of all momenta of strongly degenerate reacting fermions to the corresponding Fermi momenta $\left(\left|\boldsymbol{p}_{j}\right|=p_{\mathrm{F} j}\right)$ and values of energies of these particles to the corresponding chemical potentials $\left(\epsilon_{j}=\mu_{j}\right)$ in all functions of $\boldsymbol{p}_{j}$ and $\epsilon_{j}$ which vary smoothly within thermal energy widths in local elements near respective Fermi surfaces. Then in Eq. (3) one can set $\mathrm{d} \boldsymbol{p}_{j}=p_{\mathrm{F} j} m_{j}^{*} \mathrm{~d} \epsilon_{j} \mathrm{~d} \Omega_{j}$, where $m_{j}^{*}$ is the Landau effective mass of a fermion $j$ at the Fermi surface, and $\mathrm{d} \Omega_{j}$ is a solid angle element in the direction of $\boldsymbol{p}_{j}$. The integration over particle momenta in Eq. (1) is then decomposed into the integration over energies $\mathrm{d} \epsilon_{j}$ and over solid angles $\mathrm{d} \Omega_{j}$.

In further calculations of the emisivity $Q$ one often approximates $\mathcal{M}_{\mathcal{F} \mathcal{I}}$ by its value $\left\langle\mathcal{M}_{\mathcal{F} \mathcal{I}}\right\rangle$ averaged over orientations of particle momenta. Then the emissivity becomes

$Q=I_{\epsilon} I_{\Omega}$

where

$I_{\Omega}=\int \delta\left(\boldsymbol{P}_{\mathcal{F}}-\boldsymbol{P}_{\mathcal{I}}\right) \prod_{j} \mathrm{~d} \Omega_{j}$ is the integral over orientations of all particle momenta placed on respective Fermi surfaces, while $I_{\epsilon}$ contains all other terms (including $\left\langle\mathcal{M}_{\mathcal{F} \mathcal{I}}\right\rangle$ ) and integration over particle energies.

In a neutron star, generated neutrinos have much lower energies and momenta than the particles of the matter; it is quite sufficient to neglect neutrino momenta in the momentum-conserving delta function in Eq. (5) (e.g., Yakovlev et al. 2001). Then the integration over orientations of neutrino momentum is trivial (e.g., gives a factor of $4 \pi$ for an emission of one neutrino) and will be supposed to be included in $I_{\epsilon}$. Accordingly, the angular integration in $I_{\Omega}$ is performed only over orientations of momenta of strongly degenerate fermions $j$ of the matter. The number of these fermions will be denoted by $n$, so that $j=1, \ldots, n$ in $I_{\Omega}$.

The case of strongly interacting fermions (nucleons and hyperons in dense nuclear matter) deserves a comment. We assume that the system is non-superfluid, i.e., it is a normal Fermi liquid (see, e.g, Baym and Pethick 2007; Lifshitz and Pitaevskii 1980). Then the one-particle states with well defined energies and momenta refer actually to elementary excitations, called Landau quasiparticles. In a strongly degenerate Fermi liquid, quasiparticles form a dilute Fermi gas. Therefore, their distribution in momentum space can be well approximated by the Fermi-Dirac one. The Fermi momenta for quasiparticles coincide with those for real particles. These properties justify the use of Eq. (2). In what follows, by particles in Fermi liquids of nucleons and hyperons we will mean quasiparticles.

Systems of strongly degenerate particles are also important in many branches of physics. In particular, we can mention solid state physics (degenerate electrons in metals and semiconductors; e.g., Ziman 1960; Kittel 1986), Fermi-liquid systems (Baym and Pethick 2007) as well as nuclear physics (symmetric nuclear matter in atomic nuclei).

It is our aim to consider the angular integrals $I_{\Omega}$ for reactions involving different particle species with various Fermi momenta. These integrals determine the area of a hypersurface in $3 n$-dimensional momentum space which contributes to a given reaction. The advantage of the integrals $I_{\Omega}$ is that they are independent of specific interparticle interactions. They depend only on the total number $n$ of reacting particles and on Fermi momenta of these particles, $p_{\mathrm{F} j} \equiv p_{j}(j=1, \ldots n)$. For simplicity, we drop the subscript $\mathrm{F}$ because all momenta are assumed to be on the Fermi surfaces. The angular integrals $I_{\Omega}$ appear in many problems of Fermi systems (e.g., Ziman 1960; Baym and Pethick 2007; Shapiro and Teukolsky 1983). Some approaches for calculating them 
are described, for instance by Shapiro and Teukolsky (1983).

However, there are plenty of cases realized for different Fermi momenta. Our aim is practical, to present $I_{\Omega}$ for all possible cases at $n \leq 5$. In particular, these cases correspond to major neutrino reactions in nucleon-hyperon matter of neutron stars.

Section 2 outlines a general method for calculating $I_{\Omega}$. Sections 3, 4 and 5 present the calculations for $n \leq 5$. Applications for neutrino reactions are briefly discussed in Section 6, and we conclude in Section 7.

\section{General remarks}

Let us formulate some general properties of the angular integrals $I_{\Omega}$ as functions of $n$ Fermi momenta. Since we integrate over all possible orientations of $\boldsymbol{p}_{j}$, any inversion $\boldsymbol{p}_{j} \rightarrow-\boldsymbol{p}_{j}$ does not change $I_{\Omega}$. Therefore,

$$
\begin{aligned}
I_{\Omega} & =I_{\Omega}^{(n)}\left(p_{1}, \ldots, p_{n}\right) \\
& =\int \delta\left(\boldsymbol{p}_{1}+\ldots+\boldsymbol{p}_{n}\right) \prod_{j=1}^{n} \mathrm{~d} \Omega_{j} .
\end{aligned}
$$

This will be our starting expression. The lengths of Fermi momenta, $p_{1}, \ldots, p_{n}$, are treated as given numbers; physical nature of particle species $j$ is of no importance. For the sake of convenience, we enumerate the particles in the order of decreasing Fermi momenta,

$p_{1} \geq p_{2} \geq p_{3} \geq \ldots \geq p_{n}$.

Because the delta function in Eq. (6) describes momentum conservation, any angular integral is non-zero $\left(I_{\Omega}>0\right)$ if at least

$p_{1} \leq p_{2}+\ldots+p_{n}$.

In addition to integrals (6) it is often convenient to introduce similar auxiliary integrals

$$
\begin{aligned}
\widetilde{I}_{\Omega} & =\widetilde{I}_{\Omega}^{(n)}\left(p_{1}, \ldots, p_{n}, q\right) \\
& =\int \delta\left(\boldsymbol{p}_{1}+\ldots+\boldsymbol{p}_{n}+\boldsymbol{q}\right) \prod_{j=1}^{n} \mathrm{~d} \Omega_{j},
\end{aligned}
$$

where $\boldsymbol{q}$ is a constant vector of arbitrary length limited by momentum conservation. Because of isotropy of momentum space, $\widetilde{I}_{\Omega}$ depends only on $q=|\boldsymbol{q}|$ (but not on the direction of $\boldsymbol{q}$ ). Using the definition (6) one can present Eq. (9) in the form

$\widetilde{I}_{\Omega}^{(n)}\left(p_{1}, \ldots, p_{n}, q\right)=\frac{1}{4 \pi} I_{\Omega}^{(n+1)}\left(p_{1}, \ldots, p_{n} ; q\right)$.
It is easy to show that

$$
\begin{aligned}
& I_{\Omega}^{(n)}\left(p_{1}, \ldots, p_{n}\right) \\
& =\int \mathrm{d} \boldsymbol{q} \widetilde{I}_{\Omega}^{(m)}\left(p_{1}, \ldots, p_{m}, q\right) \widetilde{I}_{\Omega}^{(n-m)}\left(p_{m+1}, \ldots, p_{n}, q\right) \\
& =\frac{1}{4 \pi} \int q^{2} \mathrm{~d} q I_{\Omega}^{(m+1)}\left(p_{1}, \ldots, p_{m} ; q\right) \\
& \quad \times I_{\Omega}^{(n-m+1)}\left(p_{m+1}, \ldots, p_{n} ; q\right),
\end{aligned}
$$

with $m<n$. This equality greatly simplifies calculations of angular integrals because it allows one to consider the reacting particles as two subsystems $(1, \ldots, m)$ and $(m+1, \ldots, n)$. Then one can take auxiliary integrals for these subsystems separately, which is simpler than calculate $I_{\Omega}^{(n)}$ directly. In this case $\boldsymbol{q}$ is a momentum transfer from one subsystem to the other. A partition of particles into these subsystems is arbitrary. The resulting angular integral $I_{\Omega}^{(n)}$ is, of course, independent of specific partition.

Let us consider $I_{\Omega}^{(n)}$ with $n \leq 5$.

\section{Reactions involving $n=2$ and 3 fermions}

If $n=2$ we have $\boldsymbol{p}_{1}=-\boldsymbol{p}_{2}$ and Eq. (6) yields

$I_{\Omega}^{(2)}\left(p_{1}, p_{2}\right)=\frac{2(2 \pi)}{p_{1} p_{2}} \delta\left(p_{1}-p_{2}\right)$.

In the case of $n=3$ and $p_{1}<p_{2}+p_{3}$ (standard triangle condition) one obtains

$I_{\Omega}^{(3)}\left(p_{1}, p_{2}, p_{3}\right)=\frac{2(2 \pi)^{2}}{p_{1} p_{2} p_{3}}$.

It is the basic expression to be used for all angular integrals with $n>3$.

\section{Reactions involving $n=4$ fermions}

In the case of four reacting fermions one can use Eq. (11) and divide the system of four particles into two subsystems, say $(1,2)$ and $(3,4)$. Then using Eq. (13) one comes to a general expression $\left(p_{1}<p_{2}+p_{3}+p_{4}\right)$

$$
\begin{aligned}
& I_{\Omega}^{(4)}\left(p_{1}, p_{2}, p_{3}, p_{4}\right) \\
& =\frac{1}{4 \pi} \int q^{2} \mathrm{~d} q I_{\Omega}^{(3)}\left(p_{1}, p_{2} ; q\right) I_{\Omega}^{(3)}\left(p_{3}, p_{4} ; q\right) \\
& =\frac{2(2 \pi)^{3}}{p_{1} p_{2} p_{3} p_{4}} Q^{(4)}, \quad Q^{(4)}=q_{\max }-q_{\min }
\end{aligned}
$$

where $q_{\max }=p_{3}+p_{4}, q_{\min }=\max \left(p_{1}-p_{2}, p_{3}-p_{4}\right)$; $q_{\max }$ is the maximum momentum transferred between 
subsystems $(1,2)$ and $(3,4) ; q_{\min }$ is the minimum momentum transfer between these subsystems.

In the general case of $p_{1} \geq p_{2} \geq p_{3} \geq p_{4}$ we have

$$
Q^{(4)}= \begin{cases}p_{2}+p_{3}+p_{4}-p_{1} & \text { at } p_{1}+p_{4} \geq p_{2}+p_{3} \\ 2 p_{4} & \text { at } p_{1}+p_{4}<p_{2}+p_{3}\end{cases}
$$

This expression is further simplified in several particular cases listed in Table 1 - for two equal fermion momenta out of four, two different pairs of equal momenta, three equal momenta, and for equal momenta of all four fermions.

\section{Reactions involving $n=5$ fermions}

In the case of five reacting fermions one also can use Eq. (11) and divide fermions into two subsystems, for instance, $(1,2)$ and $(3,4,5)$, with $p_{1} \leq p_{2}+p_{3}+p_{4}+p_{5}$ and $p_{1} \geq p_{2} \geq p_{3} \geq p_{4} \geq p_{5}$. In this way we obtain

$$
\begin{aligned}
& I_{\Omega}^{(5)}\left(p_{1}, p_{2}, p_{3}, p_{4}, p_{5}\right) \\
& =\frac{1}{4 \pi} \int q^{2} \mathrm{~d} q I_{\Omega}^{(3)}\left(p_{1}, p_{2} ; q\right) I_{\Omega}^{(4)}\left(p_{3}, p_{4}, p_{5} ; q\right) .
\end{aligned}
$$

Then, following Eq. (14), one can introduce the second auxiliary momentum $\boldsymbol{k}$. It occurs due to an additional degree of freedom associated with the third particle in the subsystem $(3,4,5)$. Using again Eq. (13) we obtain

$$
\begin{aligned}
& I_{\Omega}^{(5)}\left(p_{1}, p_{2}, p_{3}, p_{4}, p_{5}\right) \\
& =\frac{1}{(4 \pi)^{2}} \int q^{2} \mathrm{~d} q \int k^{2} \mathrm{~d} k I_{\Omega}^{(3)}\left(p_{1}, p_{2} ; q\right) I_{\Omega}^{(3)}\left(p_{3} ; q, k\right) \\
& \times I_{\Omega}^{(3)}\left(p_{4}, p_{5} ; k\right)=\frac{2(2 \pi)^{4}}{p_{1} p_{2} p_{3} p_{4} p_{5}} Q^{(5)},
\end{aligned}
$$

where

$Q^{(5)}=\int_{\Sigma} \mathrm{d} q \mathrm{~d} k=\Sigma$,

Table 1 Particular cases of $Q^{(4)}$ for 4 fermions

\begin{tabular}{ll}
\hline \hline Conditions & $Q^{(4)}$ \\
\hline$p_{1}=p_{2}>p_{3}>p_{4}$ & $2 p_{4}$ \\
$p_{2}=p_{3}, \quad p_{1}+p_{4}>2 p_{2}$ & $2 p_{2}+p_{4}-p_{1}$ \\
$p_{2}=p_{3}, \quad p_{1}+p_{4} \leq 2 p_{2}$ & $2 p_{4}$ \\
$p_{1}>p_{2}>p_{3}=p_{4}$ & $p_{2}+2 p_{3}-p_{1}$ \\
$p_{1}=p_{2}>p_{3}=p_{4}$ & $2 p_{3}$ \\
$p_{1}=p_{2}=p_{3}>p_{4}$ & $2 p_{4}$ \\
$p_{1}>p_{2}=p_{3}=p_{4}$ & $3 p_{4}-p_{1}$ \\
$p_{1}=p_{2}=p_{3}=p_{4}$ & $2 p_{1}$ \\
\hline \hline
\end{tabular}

with $\boldsymbol{k}=\boldsymbol{p}_{4}+\boldsymbol{p}_{5}$ and $\boldsymbol{q}=\boldsymbol{p}_{3}+\boldsymbol{k}$. Therefore, $Q^{(5)}$ is equal to the area $\Sigma$ in the $(q, k)$-plane (Fig. 1) restricted by the conditions

$p_{1}-p_{2}<q<p_{1}+p_{2} \quad$ for fermions $(1,2)$;

$\left|k-p_{3}\right|<q<p_{3}+k, \quad p_{4}-p_{5}<k<p_{4}+p_{5}$

for fermions $(3,4,5)$.

The required area $Q^{(5)}$ in the $(q, k)$ plane can be calculated from a simple geometrical consideration (Fig. 1). Generally, one can distinguish six different cases which we denote as cases 1, 2, 3, 4A, 4B and 5. Geometry for these cases is presented in Figs. 2 and 3. The conditions for the realization of these cases and relevant expressions for $Q^{(5)}$ are listed in Table 2. In Fig. 2 we do not show the region of low $q$ because the area $Q^{(5)}$ lies above that region. In all the cases $1-4$ the line $q=p_{1}+p_{2}$ is placed too high and does not affect the value of $Q^{(5)}$; accordingly, we do not plot this line in such cases.

We have enumerated six cases in Table 2, in descending order in $p_{1}$, which is seen from column 2. Replacing the inequalities written in this column by the equalities we obtain the boundaries of corresponding regions in the allowed parameter space of $p_{1}, \ldots p_{5}$. The six regions fully cover the allowed parameter space, and $Q^{(5)}$ changes continuously while going from one region to another. The two subcases, $4 \mathrm{~A}$ and $4 \mathrm{~B}$, correspond to the conditions $p_{3}<p_{4}+p_{5}$ and $p_{3}>p_{4}+p_{5}$, respectively, where the area $\Sigma$ is calculated differently (see the left and middle panels in Fig. 3). The conditions $p_{3}<p_{4}+p_{5}$ and $p_{3}>p_{4}+p_{5}$ are not written explicitly in column 2 for the cases A and B because they are guaranteed by the inequalities written for these cases in column $2\left(p_{1} \geq p_{2}\right)$. In the case 5 one would also expect two similar subcases, A and B, but the subcase B, in which one would have $p_{1}<-p_{2}+p_{3}+p_{4}+p_{5}$, cannot be realized because it is incompatible with $p_{3}>p_{4}+p_{5}$ $\left(p_{1}+p_{2} \geq 2 p_{3}\right)$. Therefore, case 5 is essentially the same as subcase $5 \mathrm{~A}$.

Table 2 gives $Q^{(5)}$ for any system of five degenerate fermions. Furthermore, the results are simplified if several fermions have equal momenta. For the convenience of the reader we present such results in Tables 3 and 4 . Notice that, as a rule, the number of possible cases for equal momenta of fermions becomes lower (not all the cases are necessarily realized). In the simplest case of five equal momenta $p_{1}=p_{2}=p_{3}=p_{4}=p_{5}$, one has (case 5) $Q^{(5)}=\frac{5}{2} p_{1}^{2}$.

For illustration, Fig. 4 presents $Q^{(5)}$ as a function of $p_{1}$ for five combinations of $p_{j}(j=2, \ldots, 5)$. To exhibit dimensionless quantities we plot $Q^{(5)} / p_{2}^{2}$ versus 
Table 2 Values of $Q^{(5)}$ for all six cases displayed in Figs. $2-3$

\begin{tabular}{clll}
\hline \hline Case & Conditions & & $Q^{(5)}$ \\
\hline 1 & $p_{1}<p_{2}+p_{3}+p_{4}+p_{5} ;$ & $p_{1}>p_{2}+p_{3}+p_{4}-p_{5}$ & $\frac{1}{2}\left(p_{2}+p_{3}+p_{4}+p_{5}-p_{1}\right)^{2}$ \\
2 & $p_{1}<p_{2}+p_{3}+p_{4}-p_{5} ;$ & $p_{1}>p_{2}+p_{3}-p_{4}+p_{5}$ & $2 p_{5}\left(p_{2}+p_{3}+p_{4}-p_{1}\right)$ \\
3 & $p_{1}<p_{2}+p_{3}-p_{4}+p_{5} ;$ & $p_{1}>p_{2}+\left|p_{3}-p_{4}-p_{5}\right|$ & $4 p_{4} p_{5}-\frac{1}{2}\left(p_{1}+p_{4}+p_{5}-p_{2}-p_{3}\right)^{2}$ \\
$4 \mathrm{~A}$ & $p_{1}<p_{2}-p_{3}+p_{4}+p_{5} ;$ & $p_{1}>-p_{2}+p_{3}+p_{4}+p_{5}$ & $4 p_{4} p_{5}-\left(p_{4}+p_{5}-p_{3}\right)^{2}-\left(p_{1}-p_{2}\right)^{2}$ \\
$4 \mathrm{~B}$ & $p_{1}<p_{2}+p_{3}-p_{4}-p_{5} ;$ & $p_{1}>-p_{2}+p_{3}+p_{4}+p_{5}$ & $4 p_{4} p_{5}$ \\
5 & $p_{1}<-p_{2}+p_{3}+p_{4}+p_{5} ;$ & $p_{1} \geq p_{2}$ & $4 p_{4} p_{5}-\left(p_{4}+p_{5}-p_{3}\right)^{2}-\left(p_{1}-p_{2}\right)^{2}$ \\
& & & $-\frac{1}{2}\left(p_{3}+p_{4}+p_{5}-p_{1}-p_{2}\right)^{2}$ \\
\hline \hline
\end{tabular}

Table 3 Values of $Q^{(5)}$ for two equal fermions

\begin{tabular}{|c|c|c|}
\hline Case & Conditions & $Q^{(5)}$ \\
\hline $4 \mathrm{~A}$ & $p_{1}=p_{2}, \quad p_{3}<p_{4}+p_{5}, 2 p_{1}>p_{3}+p_{4}+p_{5}$ & $4 p_{4} p_{5}-\left(p_{4}+p_{5}-p_{3}\right)^{2}$ \\
\hline $4 \mathrm{~B}$ & $p_{1}=p_{2}, \quad p_{3}>p_{4}+p_{5}, 2 p_{1}>p_{3}+p_{4}+p_{5}$ & $4 p_{4} p_{5}$ \\
\hline 5 & $p_{1}=p_{2}, \quad 2 p_{1}<p_{3}+p_{4}+p_{5}$ & $\begin{array}{l}4 p_{4} p_{5}-\left(p_{4}+p_{5}-p_{3}\right)^{2} \\
-\frac{1}{2}\left(p_{3}+p_{4}+p_{5}-2 p_{1}\right)^{2}\end{array}$ \\
\hline 1 & $p_{2}=p_{3}, \quad p_{1}<2 p_{2}+p_{4}+p_{5}, p_{1}>2 p_{2}+p_{4}-p_{5}$ & $\frac{1}{2}\left(2 p_{2}+p_{4}+p_{5}-p_{1}\right)^{2}$ \\
\hline 2 & $p_{2}=p_{3}, \quad p_{1}<2 p_{2}+p_{4}-p_{5}, p_{1}>2 p_{2}-p_{4}+p_{5}$ & $2 p_{5}\left(2 p_{2}+p_{4}-p_{1}\right)$ \\
\hline 3 & $p_{2}=p_{3}, \quad p_{1}<2 p_{2}-p_{4}+p_{5}, p_{1}>p_{2}+\left|p_{2}-p_{4}-p_{5}\right|$ & $4 p_{4} p_{5}-\frac{1}{2}\left(p_{1}+p_{4}+p_{5}-2 p_{2}\right)^{2}$ \\
\hline $4 \mathrm{~B}$ & $p_{2}=p_{3}, \quad p_{1}<2 p_{2}-p_{4}-p_{5}, p_{1}>p_{4}+p_{5}$ & $4 p_{4} p_{5}$ \\
\hline 5 & $p_{2}=p_{3}, \quad p_{1}<p_{4}+p_{5}$ & $\begin{array}{l}4 p_{4} p_{5}-\left(p_{4}+p_{5}-p_{2}\right)^{2}-\left(p_{1}-p_{2}\right)^{2} \\
-\frac{1}{2}\left(p_{4}+p_{5}-p_{1}\right)^{2}\end{array}$ \\
\hline 1 & $p_{3}=p_{4}, \quad p_{1}<p_{2}+2 p_{3}+p_{5}, \quad p_{1}>p_{2}+2 p_{3}-p_{5}$ & $\frac{1}{2}\left(p_{2}+2 p_{3}+p_{5}-p_{1}\right)^{2}$ \\
\hline 2 & $p_{3}=p_{4}, \quad p_{1}<p_{2}+2 p_{3}-p_{5}, p_{1}>p_{2}+p_{5}$ & $2 p_{5}\left(p_{2}+2 p_{3}-p_{1}\right)$ \\
\hline $4 \mathrm{~A}$ & $p_{3}=p_{4}, \quad p_{1}<p_{2}+p_{5}, p_{1}>-p_{2}+2 p_{3}+p_{5}$ & $4 p_{3} p_{5}-p_{5}^{2}-\left(p_{1}-p_{2}\right)^{2}$ \\
\hline 5 & $p_{3}=p_{4}, \quad p_{1}<-p_{2}+2 p_{3}+p_{5}$ & $4 p_{3} p_{5}-p_{5}^{2}-\left(p_{1}-p_{2}\right)^{2}-\frac{1}{2}\left(2 p_{3}+p_{5}-p_{1}-p_{2}\right)^{2}$ \\
\hline 1 & $p_{4}=p_{5}, \quad p_{1}<p_{2}+p_{3}+2 p_{4}, p_{1}>p_{2}+p_{3}$ & $\frac{1}{2}\left(p_{2}+p_{3}+2 p_{4}-p_{1}\right)^{2}$ \\
\hline 3 & $p_{4}=p_{5}, \quad p_{1}<p_{2}+p_{3}, \quad p_{1}>p_{2}+\left|p_{3}-2 p_{4}\right|$ & $4 p_{4}^{2}-\frac{1}{2}\left(p_{1}+2 p_{4}-p_{2}-p_{3}\right)^{2}$ \\
\hline $4 \mathrm{~A}$ & $p_{4}=p_{5}, \quad p_{1}<p_{2}-p_{3}+2 p_{4}, \quad p_{1}>-p_{2}+p_{3}+2 p_{4}$ & $4 p_{4}^{2}-\left(2 p_{4}-p_{3}\right)^{2}-\left(p_{1}-p_{2}\right)^{2}$ \\
\hline $4 \mathrm{~B}$ & $p_{4}=p_{5}, \quad p_{1}<p_{2}+p_{3}-2 p_{4}, p_{1}>-p_{2}+p_{3}+2 p_{4}$ & $4 p_{4}^{2}$ \\
\hline 5 & $p_{4}=p_{5}, \quad p_{1}<-p_{2}+p_{3}+2 p_{4}$ & $\begin{array}{l}4 p_{4}^{2}-\left(2 p_{4}-p_{3}\right)^{2}-\left(p_{1}-p_{2}\right)^{2} \\
-\frac{1}{2}\left(p_{3}+2 p_{4}-p_{1}-p_{2}\right)^{2}\end{array}$ \\
\hline
\end{tabular}



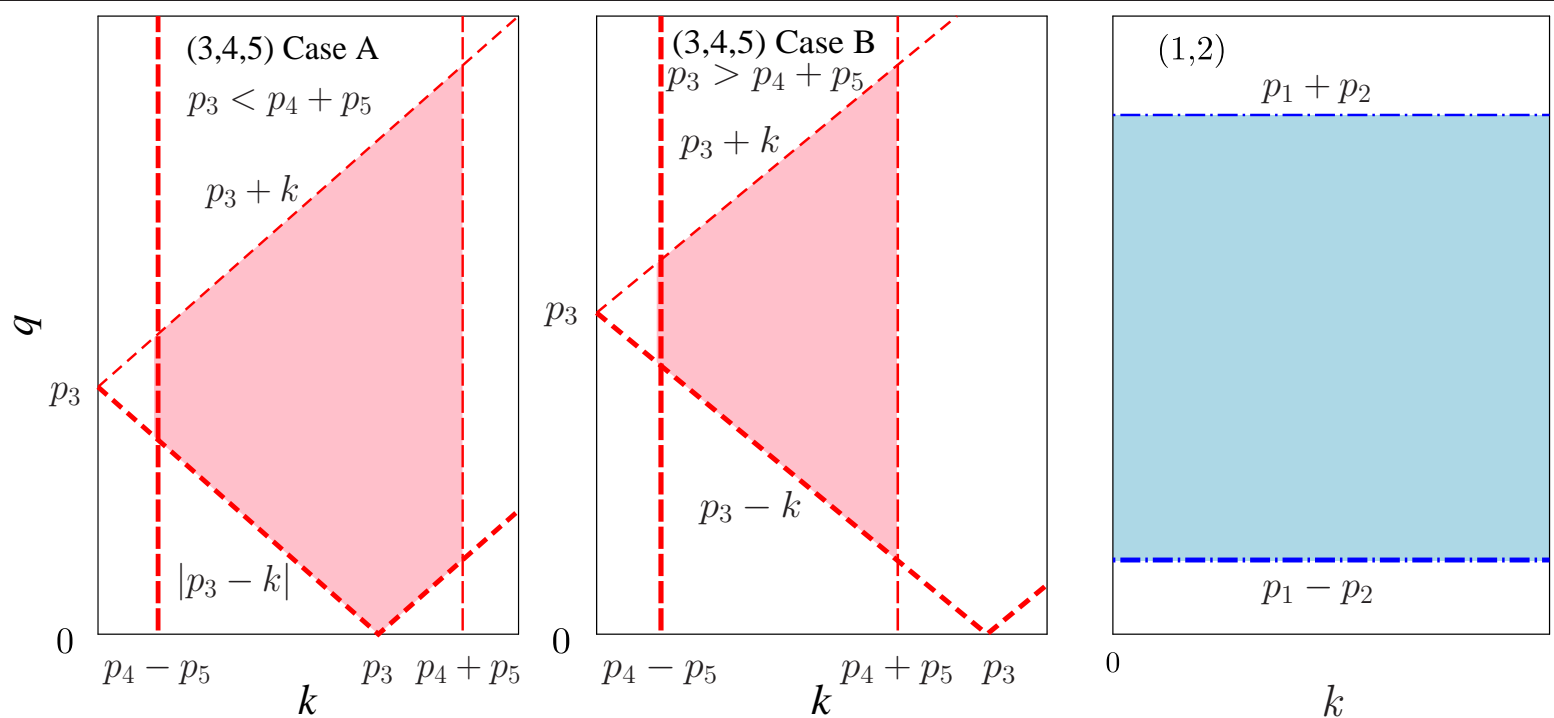

Fig. 1 Schematic representation of dashed areas in the $q-k$ plane restricted by momentum transfers in subsystem $(3,4,5)$ at $p_{3}<p_{4}+p_{5}$ (left, case A) and at $p_{3}>p_{4}+p_{5}$ (middle, case B), as well as in subsystem $(1,2)$ (right). $Q^{(5)}$ is the intersection area $\Sigma$ for the subsystems $(3,4,5)$ and $(1,2)$. In this and other figures thin short-dashed lines refer to $q=p_{3}+k$; thick short-dashed lines to $q=\left|p_{3}-k\right|$; thick long-dashed lines to $k=p_{4}-p_{5}$, thin long-dashed lines to $k=p_{4}+p_{5}$; thick dot-dashed line to $q=p_{1}-p_{2}$, while thin dot-dashed line to $q=p_{1}+p_{2}$.
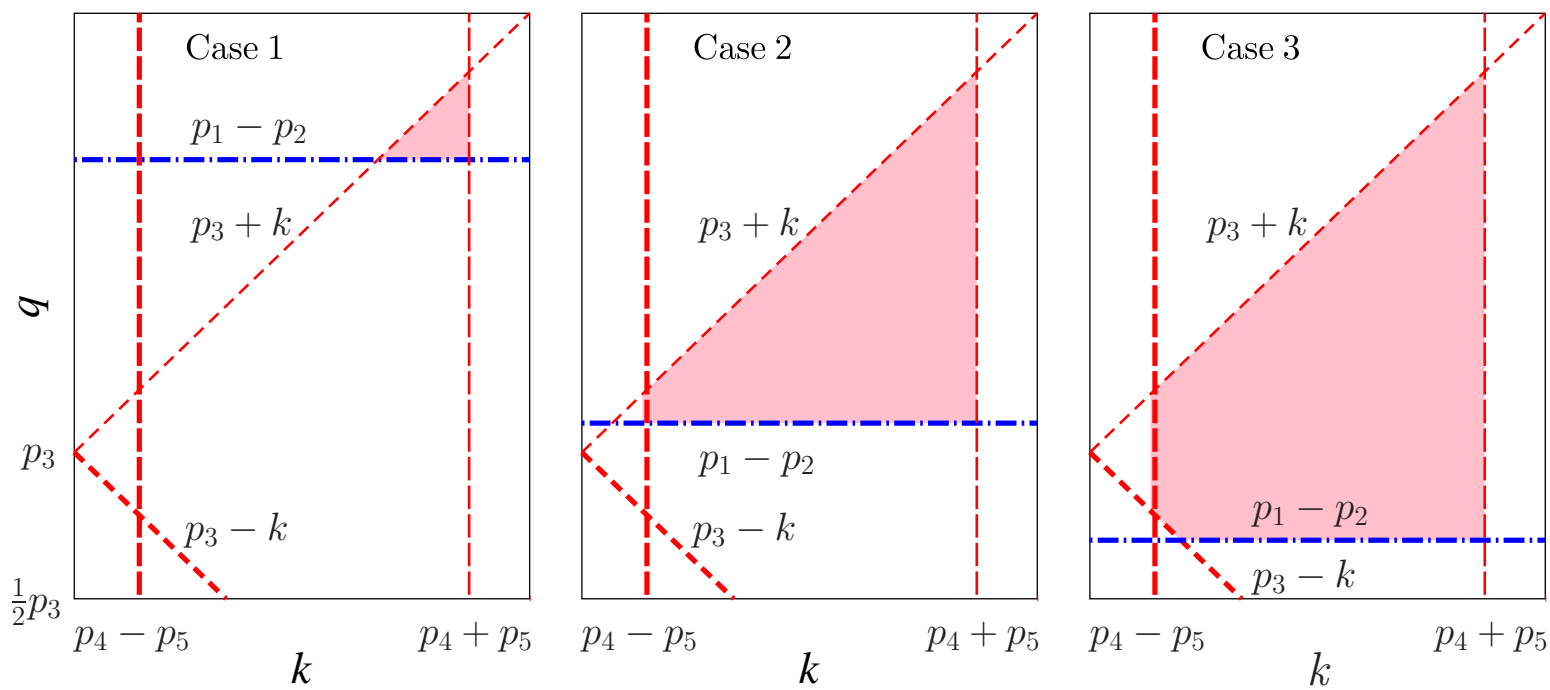

Fig. 2 Schematic representation of (shaded) areas $Q^{(5)}=\Sigma$ for cases 1,2 and 3.

$p_{1} / p_{2}$ (five lines of different types). Any line refers to a certain combination of $p_{j} / p_{2}$ listed in the table that is inserted in the figure. For the convenience of presentation, the values of $p_{j}$ are chosen in such a way that $p_{1 \max }=p_{2}+p_{3}+p_{4}+p_{5}=2.5 p_{2}$ for each combination, so that $p_{1}$ varies from $p_{2}$ to $2.5 p_{2}$. The solid, dotted and short-dashed lines correspond to the cases in which all $p_{2}, \ldots, p_{4}$ are different. The long-dashed line is for a pair of equal momenta $\left(p_{4}=p_{5}=0.35 p_{2}\right)$, while the dot-dashed line is for three equal momenta $\left(p_{3}=p_{4}=p_{5}=0.5 p_{2}\right)$. Naturally, $Q^{(5)} \rightarrow 0$ as $p_{1} \rightarrow p_{1 \max }$ for all lines (case 1 ). When $p_{1}$ decreases from $p_{1 \text { max }}$, the quantity $Q^{(5)}$ grows up owing to the increase of possible configurations of particle momenta allowed by momentum conservation.

Using similar technique one can calculate angular integrals $Q_{\Omega}^{(n)}$ at higher $n>5$. With increasing $n$ the number of different cases will be progressively larger.

\section{Discussion}

Let us outline some applications of our results. A very rich spectrum of applications is provided by neutrino 

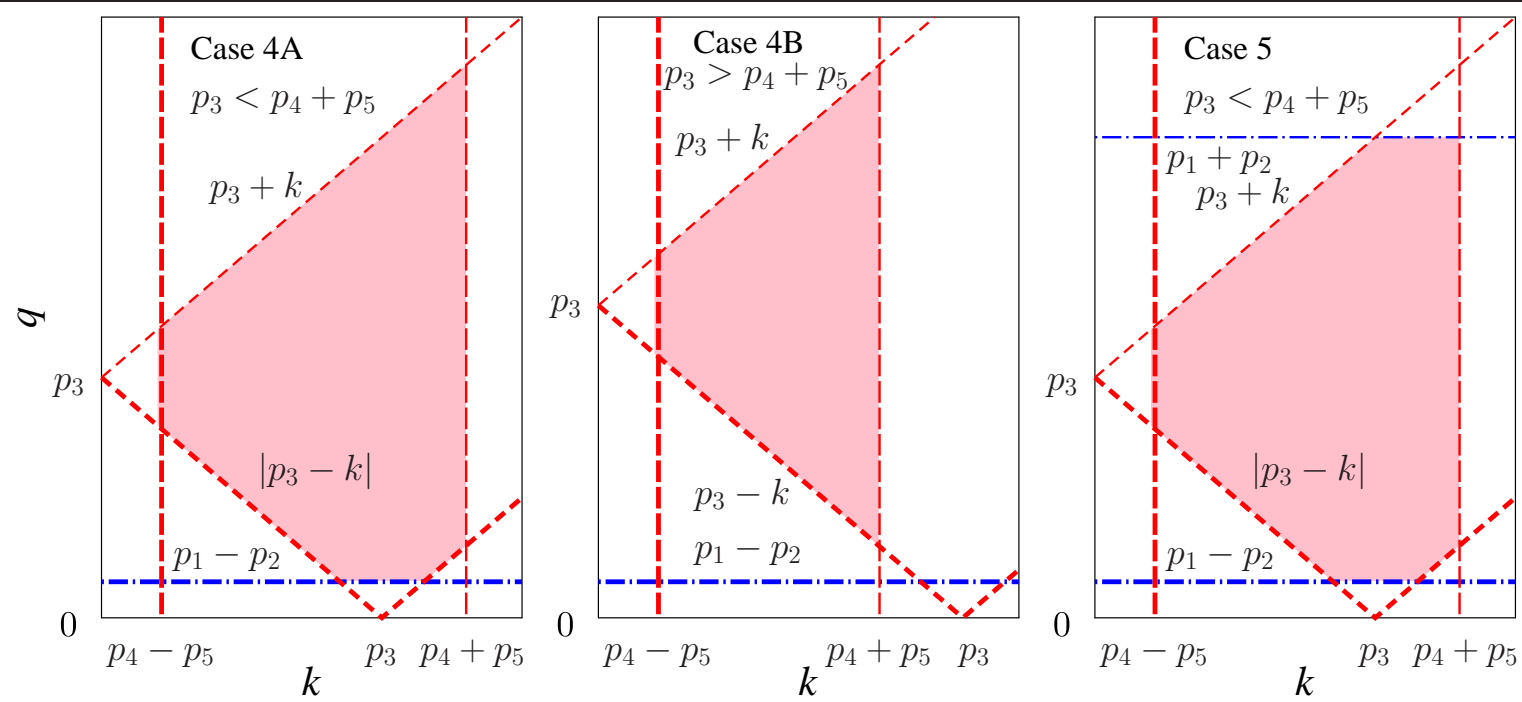

Fig. 3 Schematic representation of areas $Q^{(5)}=\Sigma$ for cases $4 \mathrm{~A}, 4 \mathrm{~B}$ and 5 .

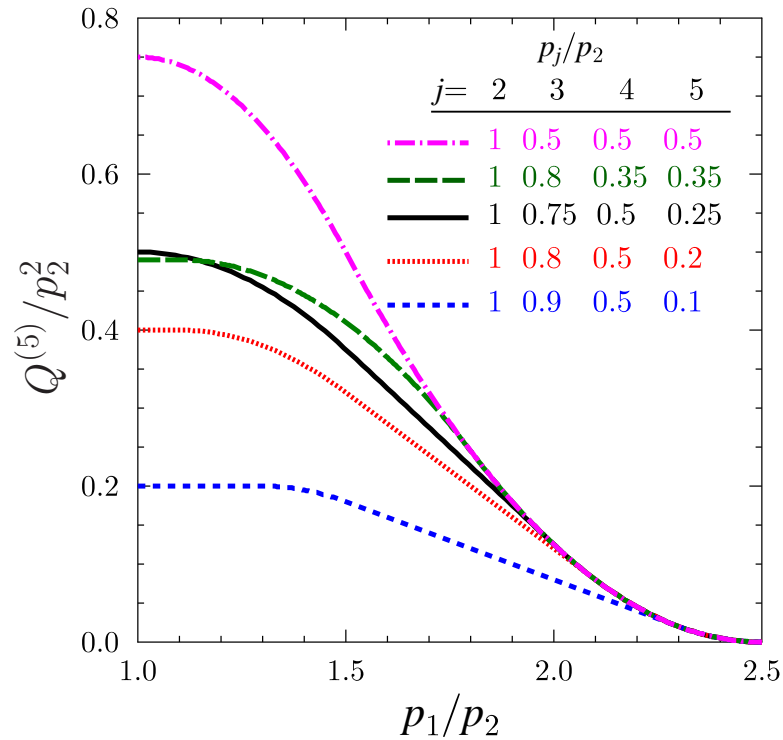

Fig. 4 Dependence of $Q^{(5)} / p_{2}^{2}$ on $p_{1} / p_{2}$ (five lines of different types) for five different combinations of $p_{2}, \ldots, p_{5}$ indicated in the inserted Table; $p_{1 \max }=2.5 p_{2}$ for all combinations (see text for details).

physics of neutron star cores (Section 1) where many neutrino mechanisms can operate and regulate thermal evolution of these stars (e.g., Yakovlev et al. 2001). The neutrino emissivities of many of these reactions, especially in nucleon-hyperon matter, have not been calculated with sufficient accuracy. The detailed calculations require the above results.

The major neutrino processes in neutron star cores can be divided into (i) direct Urca processes, (ii) baryon-baryon bremsstrahlung processes, and (iii) modified Urca processes. The approximation of angle- independent squared matrix elements (Section 1) for these reactions is usually valid (Yakovlev et al. 2001) which justifies our analysis. Direct Urca processes are much stronger, than other ones, but have a threshold character (e.g. Lattimer et al. 1991; Prakash et al. 1992). Any direct Urca process switches on once the density exceeds some threshold value, $\rho=\rho_{\text {th }}$, determined by a given process and a given equation of state of the matter. As a rule, threshold densities $\rho_{\text {th }}$ lie in the inner cores of massive neutron stars. If the direct Urca processes are allowed, the modified Urca and the bremsstrahlung processes are insignificant. Some equations of state forbid the onset of direct Urca processes at any density of matter in stable neutron stars.

Following Yakovlev et al. (2001), we illustrate the variety of neutrino emission mechanisms in a neutron star core using a model of nucleon-hyperon matter as an example and assuming the presence of $\Lambda$ and $\Sigma^{-}$hyperons. Generally, the Fermi momenta of constituents of the matter (n, p, e, $\mu, \Lambda, \Sigma)$ may be rather arbitrary (e.g., see Haensel et al. 2007 and references therein). For simplicity, we consider the baryons (n, p, $\Lambda, \Sigma)$ as non-superfluid and assume the presence of beta equilibrium and electric neutrality (Shapiro and Teukolsky 1983; Haensel et al. 2007).

\subsection{Direct Urca processes $(n=3)$}

Any direct Urca process is a sequence of two reactions,

$B_{a} \rightarrow B_{b}+\ell+\bar{\nu}_{\ell}, \quad B_{b}+\ell \rightarrow B_{a}+\nu_{\ell}$,

which results in the emission a neutrino pair. Here, $\ell$ stands for a lepton (e or $\mu) ; \nu_{\ell}$ and $\bar{\nu}_{\ell}$ are associated neutrino and anti-neutrino, respectively; $B_{a}$ and $B_{b}$ are 
Table 4 Values of $Q^{(5)}$ for more than two particles with equal momenta

\begin{tabular}{|c|c|c|c|}
\hline 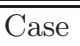 & Conditions & & $Q^{(5)}$ \\
\hline $4 \mathrm{~A}$ & $p_{1}=p_{2}, p_{3}=p_{4}$ & $2 p_{1}>2 p_{3}+p_{5}$ & $4 p_{3} p_{5}-p_{5}^{2}$ \\
\hline 5 & $p_{1}=p_{2}, p_{3}=p_{4}$ & $2 p_{1}<2 p_{3}+p_{5}$ & $4 p_{3} p_{5}-p_{5}^{2}-\frac{1}{2}\left(2 p_{3}+p_{5}-2 p_{1}\right)^{2}$ \\
\hline $4 \mathrm{~A}$ & $p_{1}=p_{2}, p_{4}=p_{5}$ & $p_{3}<2 p_{4}, 2 p_{1}>p_{3}+2 p_{4}$ & $4 p_{4}^{2}-\left(2 p_{4}-p_{3}\right)^{2}$ \\
\hline $4 \mathrm{~B}$ & $p_{1}=p_{2}, p_{4}=p_{5}$ & $p_{3}>2 p_{4}, 2 p_{1}>p_{3}+2 p_{4}$ & $4 p_{4}^{2}$ \\
\hline 5 & $p_{1}=p_{2}, p_{4}=p_{5}$ & $2 p_{1}<p_{3}+2 p_{4}$ & $4 p_{4}^{2}-\left(2 p_{4}-p_{3}\right)^{2}-\frac{1}{2}\left(p_{3}+2 p_{4}-2 p_{1}\right)^{2}$ \\
\hline 1 & $p_{2}=p_{3}, p_{4}=p_{5}$ & $p_{1}<2 p_{2}+2 p_{4}, \quad p_{1}>2 p_{2}$ & $\frac{1}{2}\left(2 p_{2}+2 p_{4}-p_{1}\right)^{2}$ \\
\hline 3 & $p_{2}=p_{3}, p_{4}=p_{5}$ & $p_{1}<2 p_{2}, p_{1}>p_{2}+\left|p_{2}-2 p_{4}\right|$ & $4 p_{4}^{2}-\frac{1}{2}\left(p_{1}+2 p_{4}-2 p_{2}\right)^{2}$ \\
\hline $4 \mathrm{~B}$ & $p_{2}=p_{3}, p_{4}=p_{5}$ & $p_{1}<2 p_{2}-2 p_{4}, \quad p_{1}>2 p_{4}$ & $4 p_{4}^{2}$ \\
\hline 5 & $p_{2}=p_{3}, p_{4}=p_{5}$ & $p_{2}<p_{1}<2 p_{4}$ & $4 p_{4}^{2}-\left(2 p_{4}-p_{2}\right)^{2}-\frac{1}{2}\left(2 p_{4}-p_{1}\right)^{2}-\left(p_{1}-p_{2}\right)^{2}$ \\
\hline $4 \mathrm{~B}$ & $p_{1}=p_{2}=p_{3}$ & $p_{1}>p_{4}+p_{5}$ & $4 p_{4} p_{5}$ \\
\hline 5 & $p_{1}=p_{2}=p_{3}$ & $p_{1}<p_{4}+p_{5}$ & $4 p_{4} p_{5}-\frac{3}{2}\left(p_{4}+p_{5}-p_{1}\right)^{2}$ \\
\hline $4 \mathrm{~B}$ & $p_{1}=p_{2}=p_{3}, p_{4}=p_{5}$ & $p_{1}>2 p_{4}$ & $4 p_{4}^{2}$ \\
\hline 5 & $p_{1}=p_{2}=p_{3}, p_{4}=p_{5}$ & $p_{1}<2 p_{4}$ & $4 p_{4}^{2}-\frac{3}{2}\left(2 p_{4}-p_{1}\right)^{2}$ \\
\hline 1 & $p_{2}=p_{3}=p_{4}$ & $p_{1}<3 p_{2}+p_{5}, \quad p_{1}>3 p_{2}-p_{5}$ & $\frac{1}{2}\left(3 p_{2}+p_{5}-p_{1}\right)^{2}$ \\
\hline 2 & $p_{2}=p_{3}=p_{4}$ & $p_{1}<3 p_{2}-p_{5}, \quad p_{1}>p_{2}+p_{5}$ & $2 p_{5}\left(3 p_{2}-p_{1}\right)$ \\
\hline 5 & $p_{2}=p_{3}=p_{4}$ & $p_{1}<p_{2}+p_{5}$ & $p_{1} p_{5}+\frac{3}{2} p_{1}\left(2 p_{2}-p_{1}\right)-\frac{3}{2}\left(p_{2}-p_{5}\right)^{2}$ \\
\hline 1 & $p_{3}=p_{4}=p_{5}$ & $p_{1}<p_{2}+3 p_{3}, p_{1}>p_{2}+p_{3}$ & $\frac{1}{2}\left(p_{2}+3 p_{3}-p_{1}\right)^{2}$ \\
\hline $4 \mathrm{~A}$ & $p_{3}=p_{4}=p_{5}$ & $p_{1}<p_{2}+p_{3}, p_{1}>-p_{2}+3 p_{3}$ & $3 p_{3}^{2}-\left(p_{1}-p_{2}\right)^{2}$ \\
\hline 5 & $p_{3}=p_{4}=p_{5}$ & $p_{1}<-p_{2}+3 p_{3}$ & $3 p_{3}^{2}-\left(p_{1}-p_{2}\right)^{2}-\frac{1}{2}\left(3 p_{3}-p_{2}-p_{1}\right)^{2}$ \\
\hline $4 \mathrm{~A}$ & $p_{1}=p_{2}>p_{3}=p_{4}=p_{5}$ & $2 p_{1}>3 p_{3}$ & $3 p_{3}^{2}$ \\
\hline 5 & $p_{1}=p_{2}>p_{3}=p_{4}=p_{5}$ & $2 p_{1}<3 p_{3}$ & $3 p_{3}^{2}-\frac{1}{2}\left(3 p_{3}-2 p_{1}\right)^{2}$ \\
\hline 1 & $p_{2}=p_{3}=p_{4}=p_{5}$ & $2 p_{2}<p_{1}<4 p_{2}$ & $\frac{1}{2}\left(4 p_{2}-p_{1}\right)^{2}$ \\
\hline 5 & $p_{2}=p_{3}=p_{4}=p_{5}$ & $p_{1}<2 p_{2}$ & $4 p_{1} p_{2}-\frac{3}{2} p_{1}^{2}$ \\
\hline 5 & $p_{1}=p_{2}=p_{3}=p_{4}$ & $p_{1}>p_{5}$ & $4 p_{1} p_{5}-\frac{3}{2} p_{5}^{2}$ \\
\hline 5 & $p_{1}=p_{2}=p_{3}=p_{4}=p_{5}$ & & $\frac{5}{2} p_{1}^{2}$ \\
\hline
\end{tabular}

baryons which undergo lepton decay or capture. In our example, we have four baryon pairs (e.g. Prakash et al. 1992) $\left(B_{a}, B_{b}\right)=(\mathrm{n}, \mathrm{p}),(\Lambda, \mathrm{p}),(\Sigma, \mathrm{n})$ and $(\Sigma, \Lambda)$, and, hence, eight direct Urca processes $(\ell=$ e or $\mu)$. The angular integrals for both reactions in (21) are evidently equal. They involve three fermions (Section 3) and are given by Eq. (13). Any direct Urca process operates as long as $p_{1} \leq p_{2}+p_{3}$, and the equality $p_{1}=p_{2}+p_{3}$ determines its threshold density $\rho_{\mathrm{th}}$. The emissivities of the direct Urca processes in the nucleon-hyperon matter have been calculated by Lattimer et al. (1991); Prakash et al. (1992).

\subsection{Bremsstrahlung processes $(n=4)$}

These are the processes of nucleon-nucleon collisions accompanied by the emission of neutrino-pairs (any flavors, $\left.\nu_{\mathrm{e}}, \nu_{\mu}, \nu_{\tau}\right)$. The angular integrals involve four fermions (Section 4). Such processes can be divided into three types (e.g. Maxwell 1987).

The bremsstrahlung processes of the first type are

$B+B \rightarrow B+B+\nu+\bar{\nu}$,

where $B=\mathrm{n}, \mathrm{p}, \Sigma$ or $\Lambda$ is any baryon (with Fermi momentum $p_{1}$ ). We have four such processes. According to the results of Section 4 , in this case

$I_{\Omega}^{(4)}=\frac{4(2 \pi)^{3}}{p_{1}^{3}}$.

The processes of the second type read

$B_{1}+B_{2} \rightarrow B_{1}+B_{2}+\nu+\bar{\nu}$,

with $p_{1}>p_{2}$. We have six such processes for which

$I_{\Omega}^{(4)}=\frac{4(2 \pi)^{3}}{p_{1}^{2} p_{2}}$.

Eqs. (23) and (25) are in line with the formulas presented in the literature (e.g., Yakovlev et al. 2001) for the nn, pp and np bremsstrahlung processes.

Finally, we have two other bremsstrahlung processes with four different fermions,

$\Lambda+\mathrm{n} \rightarrow \Sigma+\mathrm{p}+\nu+\bar{\nu}, \quad \Sigma+\mathrm{p} \rightarrow \Lambda+\mathrm{n}+\nu+\bar{\nu}$

Their angular integrals are easily calculated from Eqs. (14) and (15). They are given by different expressions depending on the values of four Fermi momenta.

The neutrino emissivities $Q$ of the bremmstrahlung processes in nucleon matter have been studied with considerable attention (e.g. Yakovlev et al. 2001 and references therein), but the emissivities of the processes 
involving hyperons are much less elaborated. Their calculation would require the angular integrals $Q^{(4)}$ of Section 4 (Table 1).

\subsection{Modified Urca processes $(n=5)$}

Modified Urca processes are similar to direct Urca ones, Eq. (21) (Section 6.1), but involve an additional baryon $B_{c}$ in the initial and final channels,

$B_{a}+B_{c} \rightarrow B_{b}+B_{c}+\ell+\bar{\nu}_{\ell}, \quad B_{b}+B_{c}+\ell \rightarrow B_{a}+B_{c}+\nu_{\ell}$.

Here, $B_{a}$ and $B_{b}$ are the same as in Eq. (21), while $B_{c}$ can be any baryon available in the matter. In our example we have $8 \times 4=32$ modified Urca processes and we need a five-fermion angular integral $I_{\Omega}^{(5)}$ for each of them. As in Section 6.1, the angular integrals for both reactions in (27) are equal. The required integrals are presented in Section 5, Tables 2-4. We can generally divide all these modified Urca processes into two groups.

In the first group we include all the processes with $B_{c}$ equal to either $B_{a}$ or $B_{b}$. These are five-fermion processes with three identical fermions. Their angular integrals are given by Eq. (17) with $Q^{(5)}$ listed in Table 4, depending on Fermi momenta of reacting particles.

The second group contains other modified Urca processes with only two identical fermions $B_{c}$ out of five. Corresponding angular integrals are also described by Eq. (17) with $Q^{(5)}$ presented in Table 3.

Consider, for instance, the simplest case of nucleon dense matter which consists of $\mathrm{n}, \mathrm{p}, \mathrm{e}$ and $\mu$. In realistic models of such a matter in neutron stars cores the neutrons dominate (the neutron number density $n_{\mathrm{n}}$ is the largest one), and the electric neutrality of the matter implies that the number densities of other particles satisfy the condition $n_{\mathrm{p}}=n_{\mathrm{e}}+n_{\mu}$. Then the Fermi momenta of the particles obey the inequality $p_{\mathrm{n}}>p_{\mathrm{p}}>p_{\ell}$. In this case we have four modified Urca processes. They are the neutron-branch $\left(\mathrm{n}+\mathrm{n} \rightarrow \mathrm{p}+\mathrm{n}+\ell+\bar{\nu}_{\ell}, \mathrm{p}+\mathrm{n}+\ell \rightarrow \mathrm{n}+\mathrm{n}+\nu_{\ell}, B_{c}=\mathrm{n}\right)$ and proton-branch $\left(\mathrm{n}+\mathrm{p} \rightarrow \mathrm{p}+\mathrm{p}+\ell+\bar{\nu}_{\ell}, \mathrm{p}+\mathrm{p}+\ell \rightarrow \mathrm{n}+\mathrm{p}+\nu_{\ell}\right.$, $\left.B_{c}=\mathrm{p}\right)$ processes with electrons or muons $(\ell=\mathrm{e}$ or $\mu)$.

Consider the neutron branch of the process using Table $4\left(p_{1}=p_{2}=p_{3}\right)$. In the case $4 \mathrm{~B}$ one has $p_{\mathrm{n}}>$ $p_{\mathrm{p}}+p_{\ell}$ meaning that the direct Urca process is forbidden (Section 6.1). Then we obtain

$I_{\Omega}^{(5)}=\frac{8(2 \pi)^{4}}{p_{\mathrm{n}}^{3}}$,

which is in line with Eq. (F.11) of Shapiro and Teukolsky (1983) or Eq. (134) of Yakovlev et al. (2001).
In the case 5 we have $p_{\mathrm{n}}<p_{\mathrm{p}}+p_{\ell}$, i.e. the direct Urca process is allowed. Then we have

$I_{\Omega}^{(5)}=\frac{8(2 \pi)^{4}}{p_{\mathrm{n}}^{3}}\left[1-\frac{3}{8} \frac{\left(p_{\mathrm{p}}+p_{\ell}-p_{\mathrm{n}}\right)^{2}}{p_{\mathrm{p}} p_{\ell}}\right]$.

However, this angular integral is rather unimportant because the modified Urca process is insignificant when the direct Urca operates.

For the proton branch of the modified Urca process we also use Table $4\left(p_{2}=p_{3}=p_{4}\right)$. In the cases 1 and 2 the condition $p_{\mathrm{n}}>p_{\mathrm{p}}+p_{\ell}$ is satisfied and the direct Urca process is forbidden. In the case 1 at $p_{\mathrm{n}}>3 p_{\mathrm{p}}-p_{\ell}$ we have

$I_{\Omega}^{(5)}=\frac{(2 \pi)^{4}}{p_{\mathrm{n}} p_{\mathrm{p}}^{3} p_{\ell}}\left(3 p_{\mathrm{p}}+p_{\ell}-p_{\mathrm{n}}\right)^{2}$,

which agrees with Eq. (141) of Yakovlev et al. (2001) but slightly differs from Eq. (29) of Haensel et al. (2001), where one factor $p_{\mathrm{p}}$ in the denominator is erroneously replaced by $p_{\mathrm{n}}$.

In the case 2 at $3 p_{\mathrm{p}}-p_{\ell}>p_{\mathrm{n}}>p_{\mathrm{p}}+p_{\ell}$ we obtain, in agreement with Eq. (35) in Haensel et al. (2002),

$I_{\Omega}^{(5)}=\frac{4(2 \pi)^{4}}{p_{\mathrm{n}} p_{\mathrm{p}}^{3}}\left(3 p_{\mathrm{p}}-p_{\mathrm{n}}\right)$.

If the direct Urca process is open, $p_{\mathrm{n}}<p_{\mathrm{p}}+p_{\ell}$, we come to the case 5 in which

$I_{\Omega}^{(5)}=\frac{2(2 \pi)^{4}}{p_{\mathrm{p}}^{3}}\left[1+\frac{3}{2} \frac{2 p_{\mathrm{p}}-p_{\mathrm{n}}}{p_{\ell}}-\frac{3}{2} \frac{\left(p_{\mathrm{p}}-p_{\ell}\right)^{2}}{p_{\mathrm{n}} p_{\ell}}\right]$.

This case, omitted in literature, is insignificant because the direct Urca process is much stronger than the modified one.

As for the numerous modified Urca processes with hyperons, their study has to be performed accurately based on the results of Section 5 .

\subsection{Other applications}

In solid-state physics (metals, degenerate semiconductors) one often needs (Ziman 1960; Kittel 1986) effective collision frequencies of strongly degenerate electrons $(\mathrm{e}+\mathrm{e} \rightarrow \mathrm{e}+\mathrm{e})$ which contain $I_{\Omega}^{(4)}$ (Section 4) with four equal Fermi momenta of strongly degenerate electrons, $p_{\mathrm{Fe}} \equiv p_{\mathrm{e}}$. These collision frequencies determine kinetic coefficients due to electron-electron collisions. Using Eq. (14) and the last line in Table 1 we immediately obtain the well known result $I_{\Omega}^{(4)}=4(2 \pi)^{3} / p_{\mathrm{e}}^{3}$ (e.g., Ziman 1960). The variety of similar applications in different studies of strongly degenerate fermionic systems is very large. 


\section{Conclusions}

We have described calculations of angular integrals $I_{\Omega}^{(n)}$, Eq. (6), which determine neutrino emissivities, reaction rates and related quantities for reactions involving $n$ degenerate fermions (in initial + final channels) with different or equal Fermi momenta $p_{1}, \ldots, p_{n}$. These angular integrals often occur in applications if differential reaction probabilities are determined by angleaveraged squared matrix elements (Section 1). The advantage of angular integrals $I_{\Omega}^{(n)}$ is that they solely depend on $n$ Fermi-momenta $p_{1}, \ldots, p_{n}$, being independent of the nature of reacting fermions and their interactions. The integrals $I_{\Omega}^{(n)}$ are described by analytic expressions which may have different forms, but they can be calculated once and forever.

We have calculated $I_{\Omega}^{(n)}$ for all possible cases with $n=2$ and 3 (Section 3), 4 (Section 4) and 5 (Section 5). The formalism we have used (Section 2) allows one to perform similar calculations for higher $n$.

In Section 6 we have outlined some applications of the results, particularly, for neutrino emission processes in neutron star cores composed of nucleons and hyperons. For illustration, we have discussed the expressions for angular integrals of major neutrino emission processes in neutron star cores containing neutrons, protons, electrons, muons, as well sigma and lambda hyperons. They are eight direct Urca processes $(n=3)$, 12 baryon-baryon bremsstrahlung processes $(n=4)$ and 32 modified Urca processes $(n=5)$. The majority of these neutrino reactions have not been studied with considerable attention. We provide the angular integrals which are the most important ingredients for such studies. Our results can be useful for constructing a uniform database of neutrino emissivities in nucleonhyperon matter of neutron star cores which is needed to simulate thermal structure and evolution of neutron stars.

Let us stress that much work is required to complete such a database. Aside of the angular integrals calculated here, one needs the matrix elements of many neutrino reactions as well as the factors which describe the suppression of these reactions by possible superfluidity of nucleons and hyperons. This suppression can be either very strong or weak depending on (largely unkown) critical temperatures for superfluidity of different particles (e.g., Yakovlev et al. 2001). It would be a complicated project to calculate the matrix elements and suppression factors from first principles but we expect to simplify this task using some selfsimilarity criteria, like those formulated, in Yakovlev et al. (2001). In addition, superfluidity of various baryon species can induce a specific neutrino emission due to Cooper pairing of baryons. Such processes involving hyperons should also be studied and included into the database taking into account in-medium effects in systems of superfluid baryons (Leinson and Pérez 2006; also see references given by Page et al. 2011; Shternin et al. 2011).

Note also, that neutrino reactions can be affected by strong magnetic fields. Much work should be done to study the effects of magnetic fields on various neutrino processes. The available calculations of these processes in magnetized neutron star crust and nucleon core (reviewed by Yakovlev et al. 2001) show that one typically needs very strong fields to affect the neutrino emission of neutron stars. For instance, as demonstrated by Baiko and Yakovlev (1999), the direct Urca process in nucleon neutron star core can be noticeably affected by the fields $B \gtrsim 10^{16} \mathrm{G}$.

The calculated angular integrals can also be used to study neutrino emissivities in quark stars and hybrid stars or study cooling properties of compact stars due to the emission of other weakly interacting particles (for instance, axions; e.g., Sedrakian 2016).

In a crust of a neutron star one can deal with neutrino reactions of atomic nuclei and degenerate electrons (e.g. Yakovlev et al. 2001; Bisnovatyi-Kogan, G. S. 2001, 2002). For instance, it can be neutrino-pair bremsstrahlung in electron-nucleus collisions or Urca cycles involving Urca pairs of atomic nuclei. In these cases the nuclei do not behave as strongly degenerate fermions and the neutrino emissivities are not directly expressed through the angular integrals $I_{\Omega}$ [although may contain similar integrals $\widetilde{I}_{\Omega}$, Eq. (9)].

The authors are indebted to P. Shternin for strong and constructive criticism and to K. Levenfish for encouragement. The work by DY has been supported partly by the RFBR (grants 14-02-00868-a and 16-2913009-ofi-m) and the work by PH by the Polish NCN research grant no. 2013/11/B/ST9/04528. One of the authors (A.D.K.) is grateful to N. Copernicus Astronomical Center in Warsaw for hospitality and perfect working conditions. 


\section{References}

Baiko, D. A. and Yakovlev, D. G.: Direct Urca process in strong magnetic fields and neutron star cooling, Astron. Astrophys., 342, 192-200, 1999.

Baym, G. and Pethick, C.: Landau Fermi-Liquid Theory: Concepts and Applications, Wiley-VCH, 2007.

Bisnovatyi-Kogan, G. S.: Stellar Physics. Vol. 1: Fundamental Concepts and Stellar Equilibrium, Springer, Berlin, 2001.

Bisnovatyi-Kogan, G. S.: Stellar Physics. Vol. 2: Stellar Evolution and Stability, Springer, Berlin, 2002.

Haensel, P., Levenfish, K. P., and Yakovlev, D. G.: Bulk viscosity in superfluid neutron star cores. II. Modified Urca processes in npe $\mu$ matter, Astron. Astrophys., 372, 130 137, 2001.

Haensel, P., Levenfish, K. P., and Yakovlev, D. G.: Bulk viscosity in superfluid neutron star cores. III. Effects of $\Sigma^{-}$hyperons, Astron. Astrophys., 381, 1080-1089, 2002.

Haensel, P., Potekhin, A. Y., and Yakovlev, D. G.: Neutron Stars. 1. Equation of State and Structure, vol. 326 of Astrophysics and Space Science Library, Springer, New York, 2007.

Kittel, C.: Quantum Theory of Solids, Wiley, New York, 1986.

Lattimer, J. M., Pethick, C. J., Prakash, M., and Haensel, P.: Direct URCA process in neutron stars, Phys. Rev. Lett., 66, 2701-2704, 1991.

Leinson, L. B. and Pérez, A.: Vector current conservation and neutrino emission from singlet-paired baryons in neutron stars, Phys. Lett. B, 683, 114-118, 2006.

Lifshitz, E. M. and Pitaevskii, L. P.: Statistical Physics. Part 2, Pergamon, Oxford, 1980.

Maxwell, O. V.: Neutrino emission processes in hyperonpopulated neutron stars, ApJ, 316, 691-707, 1987.

Page, D., Prakash, M., Lattimer J. M., and Steiner A. W.: Rapid cooling of the neutron ntar in Cassiopeia A triggered by neutron superfluidity in dense matter, PRL, 106, 081101, 2011

Prakash, M., Prakash, M., Lattimer, J. M., and Pethick, C. J.: Rapid cooling of neutron stars by hyperons and Delta isobars, ApJ, 390, L77-L80, 1992.

Sedrakian, A.: Axion cooling of neutron stars, PRD, 93, 065044, 2016.

Shapiro, S. L. and Teukolsky, S. A.: Black holes, white dwarfs, and neutron stars: The physics of compact objects, Wiley-Interscience, New York, 1983.

Shternin P. S., Yakovlev D. G., Heinke C. O., Ho W. C.G., and Patnaude D. J.: Cooling neutron star in the Cassiopeia A supernova remnant: evidence for superfluidity in the core, MNRAS, 412, L108, 2011.

Yakovlev, D. G., Kaminker, A. D., Gnedin, O. Y., and Haensel, P.: Neutrino emission from neutron stars, Phys. Rep., 354, 1-155, 2001.

Ziman, J. M.: Electrons and Phonons, Oxford at the Clarendon, Oxford, 1960.

This manuscript was prepared with the AAS LATEX macros v5.2. 